\title{
Report on Market Analysis and Preventions Need to Provide Medications for Rural Patients of Italy using ICT Technologies
}

\author{
Gopi Battineni, Nalini Chintalapudi, Francesco Amenta, Seyed Khosrow Tayebati
}

\begin{abstract}
Access to healthcare services is critical to good health, yet residents of internal areas face a variety of access barriers. The obstacles faced by health care providers and patients in rural areas are vastly different from those in urban areas. This might be caused by economic factors, cultural and social differences, educational shortcomings, lack of recognition by legislators and the sheer isolation of living in internal areas all conspire to create health care disparities and impede people living in internal areas in their struggle to lead normal healthy lives. Italy's indicators of health system outcomes and quality are consistently good. This is despite levels of health spending below other high-income countries like Germany, France, and other EU nations. However, Italy is lagging in some areas, like long-term care and prevention of non-communicable diseases. Based on available analyses, further progress is called for to promote appropriateness of care, address geographic imbalances in health care use and prevent the spread of risk factors including chronic diseases and regular health issues. To eradicate these issues, it is essential to develop a system that will help internal areas patients by using contemporary technologies. The improved system could assist patients also without the presence in the same place of a doctor that will generate corresponding prescriptions, which authenticated by physicians will be stored in the database.
\end{abstract}

Keywords: Healthcare, Rural area patients, Artificial Intelligence, Pathologies, ICT

\section{INTRODUCTION}

Maintaining access for health services is basic thing to better health, but internal area patients having number of barriers for accessing [1]. The issues that had looked by human services suppliers and patients in rustic regions are largely not quite the same as those in urban regions. It might be caused due to the economic factors, social differences, weak literacy rates, lack of government initiatives and the sheer segregation of living in provincial territories all scheme to make human services inconsistencies and hinder country Italian individuals in their battle to lead typical solid lives [1], [2].

The rural or internal area patients have persistent barriers to having medical services because of the lack of suitable transport services, financial crisis, and profound knowledge in health care. As of this, providing better health service in rural areas had become a more significant

Revised Manuscript Received on November 08, 2019.

* Correspondence Author

Gopi Battineni*, Telepharmacy and telemedicine Centre, university of camerino, Italy. Email: gopi.battineni@unicam.it

Nalini Chintalpdui, Telepharmacy and telemedicine Centre, university of camerino, Italy. Email: gopi.battineni@unicam.it

Prof. Francesco Amenta, Director of telemedicine and Telemedicine center, university of camerino, Italy. Email: francesco.amenta@unicam.it

Prof. Seyed Khosrow Tayebati, Full time professor in neuroscience at university of camerino, Italy. Email: khosrow.tayebati@unicam.it challenge for local governments, as of now Italy is the only country that has implemented policies on rural area health issues [3]. Nevertheless, having communication with outlying areas is a big task to provide adequate medical support. To overcome these problems ICT comes into the limelight by integrating information with unique communication, thanks to the advancement of artificial intelligence (AI) technologies [4].

The Italian advisers of wellbeing framework results and quality are reliably brilliant. It is regardless of levels of wellbeing spending underneath other high-pay nations like Germany, France, and other EU countries. In any case, Italy is slacking in certain zones, as long term care and counteractive action of non-transferable sicknesses. In view of accessible examinations, further progress is called for to advance appropriate consideration, and address geographic uneven characters of human services use and predict the spread of hazard variables including chronic diseases and regular health issues [5-7]. Therefore, to avoid these issues, it is essential to develop a system with help of Information and Communication Technologies (ICT) that will help rural area patients by using contemporary technologies. The improved system would assist patients without the presence of a doctor, patients will be treated automatically by entering their diagnosis/symptoms, and the system generates corresponding prescriptions, which authenticated by physicians and stored in the database.

In this paper, we try to explore the needs of ICT technologies to provision of health services in Italy. Also, we analyzed health markets trends in European countries that spend on the needs and concerns to improve health and safety education to prevent pathologies.

\section{METHODS}

\subsection{Provision of an electronic health record (EHR)}

High-quality medical care cannot be delivered without knowledge of the clinical history of the patient8. The so-called virtual medical records are an automatic tool that can provide individual access to someone health information easily and quickly, and in any mobility situation. These tools will allow sharing information with a doctor or a team of doctors in medical care (prevention, diagnosis, and treatment). With the gradual introduction of new welfare models and the evolution of ICT technologies that make electronic healthcare possible today, clinical data management has become one of the top priorities. In addition to the traditional hospitals and outpatient clinics (specialists and general practitioners), which have

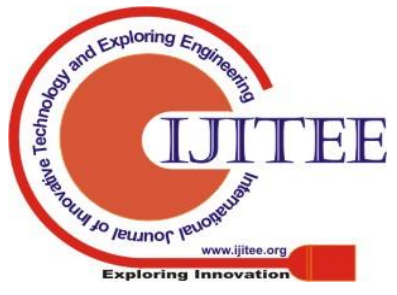


traditionally developed the electronic patient record (EPR) [8], electronic health file (EHF) [9] , or the concept of electronic health record (EHR) [10] is developing. In theory, the two types of folders differ in the scope that they refer to as the first document a cure procedure. For instance, hospital or outpatient access, while the second one collects all the primary clinical information of the patient during their life. Experimental testing will choose the EHR type and satisfaction by users at different levels of service will be a shared and accessible information tool for all those who have the structural chronological collection of events and longitudinal information. It will include the clinical history of the patient, making it a group of clinical data. Data could be used not only for occupational medicine, and also for more specific clinical purposes. With the development of extended digital health surveillance records drafted in English and Italian, the portability of health data will become a reality and will become an essential repository of patient medical information in case of medical visits or hospitalization in distant areas.

2.2. Assembling, synchronizing and interface of a range of medical equipment that can carry out at the distance health surveillance visits at rural areas

To carry out reasonable therapeutic activities at a distance is essential that medical information that comes from the periphery to the medical center was responsible for healthcare. It is supported by the receipt of objective biomedical data collected from devices installed at rural areas. This solution, which, in addition to avoiding the subjectivity of information, among other things, sent by people lacking basic medical knowledge, is in line with the guidelines of modern medicine, which is becoming more and more technological and assisted by objective instrumental evaluations.

These devices may also be used for the rural implementation of all the health surveillance activities provided for by national legislation. Moreover, these telemedical devices being used for the safety and health requirements of domestic area patients with an extraordinary improvement in diagnosis and care.

The set of instrumentation to be installed for rural site telemedicine needs is listed below
a. Infrared thermometer
b. HD Camera
c. Digital phonoscope
d. Pressure gauge12-lead electrocardiograph
e. Spiro meter
f. Oximeter
g. Video communications system

\subsection{System Testing}

The overall EHR system will be tested with large-scale experiments on local patient records. Tests will be performed for sufficient time to collect the results of simulations and any actual cases. At the end of the trial on each site, a questionnaire on the quality of this service will be proposed to the end-users of the website.

2.4. Launch procedures for the recognition of the equivalence of health surveillance activities about local visits

System prototypes will be presented to the Ministry of Infrastructure and transport with the aim of initiating the process for the recognition of equivalence of surveillance activities with the conventional ones. It should be underlined that regular surveillance is carried out with a shortage of effective system (about transport difficulties when the patient is not able to move) by a competent physician and in person. The ICT systems intend to become the leader and the economic provider of Web-based software applications in the healthcare industry.

\section{MARKET VALUES}

\subsection{Market Analysis:}

Telemedicine is experiencing some significant market growth, and a few key factors are driving it, including rising interest for available medical services for internal area patients, mechanical progressions in media transmission and restorative administrations, and an expanding need to diminish the general cost on medicinal services by the patients [11]. With higher rates in the predominance of the geriatric populace around the world, telemedicine administrations are required to experience good request in the coming years. As indicated by the latest market trends, by 2025 the worldwide telemedicine market should be about 3 billion dollars.

\subsection{Market value:}

The European e-Health market has an expected $€ 20$ billion with a yearly development of 3 percent. Thinking about the interest for medicinal services items and administrations will rise altogether in the coming years; the data innovation applied to the social insurance frameworks is significant for conveying increasingly viable and effective medical services. In Italy, the ICT consumption in social insurance in 2017 was $€ 1.3$ billion comparing to $1.1 \%$ of the overall medicinal services uses, which is not exactly numerous other advanced nations that normal $2.5-3 \%$. In spite of the $2 \%$, expansion contrasted with 2016 costs the selection of computerized instruments in medicinal services stays divided (Refer table 1).

As indicated by information, the Italian e-health consumptions over open social insurance uses will probably arrive at $1.36 \%$ in 2020 . Specialists hope to see speculations throughout the following three years in electronic wellbeing records, distributed computing, managerial administration, advanced administration of medications, E-remedy, versatile wellbeing, and business insight, and clinical administration. Table1: Expenses of E-health trends in Italy

\begin{tabular}{|c|c|c|c|c|c|}
\hline E-health & $\begin{array}{l}\text { GDP } \\
\text { in } \\
\text { million } \\
(\$)\end{array}$ & $\begin{array}{c}\text { Health } \\
\text { care } \\
\text { costs/ } \\
\text { GDP } \\
\%\end{array}$ & $\begin{array}{l}\text { Healthcare } \\
\text { costs } \\
\text { (\$) }\end{array}$ & $\begin{array}{l}\text { E-health } \\
\text { payment } \\
\mathrm{s}(\$)\end{array}$ & $\begin{array}{c}\text { e-health } \\
\text { payments/ } \\
\text { public } \\
\text { healthcare } \\
\text { expenditu } \\
\text { res \% }\end{array}$ \\
\hline 2016 & 1.671 & 6.81 & 112,386 & 1,386 & 1.25 \\
\hline 2017 & 1.715 & 6.72 & 113,787 & 1,437 & 1.27 \\
\hline 2018 & 1.764 & 6.63 & 115,155 & 1,492 & 1.31 \\
\hline 2019 & 1.818 & 6.57 & 117,466 & 1,558 & 1.35 \\
\hline 2020 & 1.87 & 6.36 & 119,215 & 1,618 & 1.39 \\
\hline
\end{tabular}




\subsection{The need for new technologies in telemedicine:}

Assessing the long term prospects of any creative methodology or innovation is a hard activity. In medicinal services, where everybody concurs the framework is parted, this assessment winds up full of the expectation that this development will be the one to correct side for internal patients. When we spot such a weight on any single change, it will be $99.9 \%$ of the time be found as need.

\section{DISCUSSION}

\subsection{Technical factors:}

Medical services is only a business opportunity for innovation where consumers like clinics are glad to pay colossal measures of cash, especially for the correct gear, for example, PET and MRI scanners and straight accelerators [12]. Quickened cost investment funds technology mechanizes and stretches out things that recently must be finished by individuals. Before implantation taps, medical attendants needed to give infusions from time to time; the imbuement tap innovation mechanized that.

Some plastic embellishment procedure will make a huge number of implantation taps as fast as it makes one; when one infusion pump has been modified in programming, it costs basically nothing to program them all. The innovation like a MRI scanner that can examine anybody similarly well will be substantially more prominent than an innovation that must be modified to a specific patient's conditions.

\subsection{Personal healthcare}

As of now, the presumptions of large scale manufacturing are evolving. For instance, the present 3D printers are fit for making objects of any shape; they are marginally less proficient than standard large scale manufacturing, however the expenses of custom objects of explicit sorts have been brought down essentially. It is presently conceivable to uniquely design titanium embeds the correct shape and size to fit. On the off chance that we can customize social insurance, we get populace measured markets: rather than offering to clinicians, makers can sell people a market 1000s of times progressively noteworthy.

\subsection{Strategies and Implementation Summary}

Development of artificial intelligence systems aimed at more excellent protection of rural area patients through the elaboration of detailed medical assistance requests

\subsubsection{The situation of medical care for rural area patients} The possibility to provide adequate medical assistance for people that living in internal areas is a challenge. The lack of healthcare centers or clinics, the limited medical knowledge of the patient and medical supplies make rural area patients in a disadvantageous condition compared to people living in urban areas.

In the case of accidents or sudden strokes, patients in rural areas had faced great difficulties in approaching doctors due to lack of proper transport or cost issues. In this uncomfortable scenario, it should be not forgotten that the appropriate use of modern telecommunications and rural medical care technologies could help very much assistance in favor of the so-called rural patients (which cannot be reached easily or in a reasonable lapse of time by healthcare professionals).

Hence, technology can overcome, often brilliantly, the handicap represented by being ill at the sea and with modest healthcare resources. The request for medical support from the patient homes to a company requires specific skills and training that, at best, are quite rudimentary. To improve teleconsulting skills, it is, therefore, necessary to make available systems that facilitate the formulation of telemedicine requests correctly also by people who have not received adequate and specific health education.

\subsubsection{Development of ICT medical care systems for rural patients}

The ICT system which needs to develop consists of easy-to-manage software that guides telemedicine assistance from patients who have no medical knowledge. In general, despite the compulsory presence in rural areas of personnel who has attended medical care or first aid training, the medical capabilities of these people are very modest.

In general, requests for telemedical advice are not detailed unless people are guided in preparing it. This makes it difficult for the physician in charge of patient's treatment to produce enough precise diagnosis and therefore to prescribe appropriate medication. Given this, the physician should ask questions, the answers to which might be imprecise, resulting in a loss of time. A simple and interactive guide may contribute to the preparation of a proper request for medical advice. This can bring a significant advantage in case of a disease or injuries allowing the rural doctor to take care properly of the patient.

\section{CONCLUSION}

In conclusion, providing telemedical assistance for rural area patients is a great challenge faced by all the European countries. On this, we firmly believe that proposed ICT solutions in this report will be work as an excellent solution to overcome all the barriers that were associated.

\section{REFERENCES}

1. K. Källander et al., "Mobile health (mhealth) approaches and lessons for increased performance and retention of community health workers in lowand middle-income countries: A review," Journal of Medical Internet Research. 2013.

2. G. Messina, F. Vencia, S. Mecheroni, S. Dionisi, L. Baragatti, and N. Nante, "Factors affecting patient satisfaction with emergency department care: an Italian rural hospital," Glob. J. Health Sci., 2015.

3. R. Papp et al., "Perceptions of quality in primary health care: Perspectives of patients and professionals based on focus group discussions," BMC Fam. Pract., 2014.

4. J. Gómez, B. Oviedo, and E. Zhuma, "Patient Monitoring System Based on Internet of Things," in Procedia Computer Science, 2016.

5. J. P. Mackenbach et al., "Socioeconomic inequalities in health in 22 European countries," N. Engl. J. Med., 2008.

6. S. Allender, P. Scarborough, V. Peto, and M. Rayner, "European cardiovascular disease statistics 2008 edition," Health Econ., 2008.

7. World Health Organisation, "Status Report on Alcohol and Health in 35 European Countries," Who, 2013.

8. V. Torchilin, "Tumor delivery of macromolecular drugs based on the EPR effect," Advanced Drug Delivery Reviews. 2011.

9. T. Peregrin, "Personal and Electronic Health Records: Sharing Nutrition Information across the Health Care Community," J. Am. Diet. Assoc., 2009.

10. A. G. Hirsch et al., "The electronic health record audit file: The patient is waiting," J. Am. Med. Informatics Assoc., 2017.

11. S. K. Mishra, L. Kapoor, and I. P. Singh, "Telemedicine in India: Current Scenario and the Future," Telemed. e-Health, 2009.

12. R. M. Warner, C. Bastianpillai, B. W. Lamb, N. Sevdalis, and J. S. A. Green, "Health services research," J. Clin. Urol., 2018.

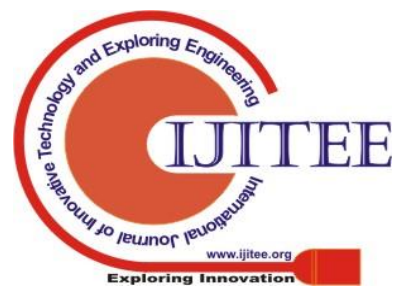




\section{AUTHORS PROFILE}

Dr. Gopi Battineni is a Ph.D. scholar at University of Camerino, Italy. He received his masters in computer science from Sheffield Hallam University, UK in 2016. His research interests are machine learning, data mining, process mining, artificial intelligence and informatics in medicine

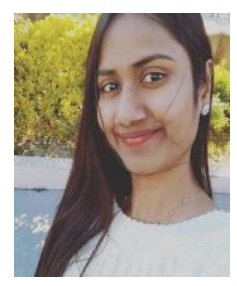

Dr. Nalini Chintalapudi is a Ph.D. scholar at University of Camerino, Italy. She received her masters in computer science from Jawaharlal Nehru Technological University, India in 2015. She is expert in database management, cloud computing, and web design

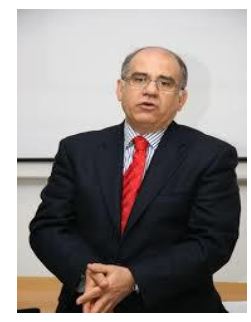

Prof. Francesco Amenta is full professor, Dean of the School of Medicinal and Health Sciences Products and Director of Telemedicine and Telepharmacy Centre of the University of Camerino, Italy. He is also the President of International Radio Medical Centre (C.I.R.M), the Italian Telemedical maritime Assistance Service and is author made publication of more than 400 research papers in the field research of pharmacology, neurology, and public health.

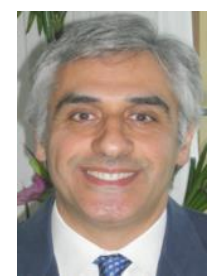

Prof. Seyed Khosrow Tayebati is associate professor of Human Anatomy at University of Camerino, Italy. His expertise is cell culture, synthesis, animal models, receptors, neurobiology, and electrophysiology. $\mathrm{He}$ is author of 79 articles in peer-reviewed international journals. 